\title{
Arterial Access for Cardiovascular Procedures
}

\author{
Jyotsna Maddury ${ }^{1}$ \\ ${ }^{1}$ Department of Cardiology, Nizams Institute of Medical Sciences, \\ Hyderabad, Telangana, India
}

Indian J Cardiovasc Dis Women-WINCARS 2018;3:264-272

\section{Introduction}

There are multiple ways by which left heart catheterization can be done. Commonly used accesses are the femoral and radial route for cardiovascular procedures. In subset of patients other than the standard routes of arterial access is required. This article is to cover mainly all of the arterial access available in present era. ${ }^{1}$

\section{Femoral Artery Access}

Femoral artery access can be done by puncturing the femoral artery either antegradely or retrogradely.

\section{Retrograde Puncture}

Usually, femoral artery puncture is done retrogradely for cardiac catheterization. Ideally, common femoral artery (CFA) should be punctured 1 to $2 \mathrm{~cm}$ below the inguinal ligament, because of two reasons: (1) This point of puncture is above the bifurcation of CFA into the superficial femoral artery (SFA) and profunda femoris; and (2) at this site, CFA is against the head of the femur. Therefore, better arterial compression can be done after the sheath removal.

\section{Technique of Puncture}

- Palpatory method: Classic way of puncturing either side of the femoral artery (FA) is by Seldinger's technique. The palpatory method is the most frequently used method for FA access. Puncture needle size of $18 \mathrm{G}$ and 0.35 -in short guidewire are used for FA accesses. The FA puncture is done in the groin where the maximal impulse is felt. Every precaution should be taken to puncture only the anterior wall of CFA, but not completely through the puncture. The CFA size is approximately 6 to $7 \mathrm{~mm}$, so puncture of the posterior wall should be avoided to prevent hematoma formation. Even though this method is frequently done, mostly we are sure of puncturing the safe place on the surface of SFA. Therefore, fluoroscopic guidance is better than palpatory method.

- Fluoroscopic guidance for femoral artery puncture: As shown in - Fig. 1, needle entry should be beneath the midline or the femoral head or inferior border of the middle neck of the femur.

\begin{abstract}
Address for correspondence Jyotsna Maddury, MD, DM, FACC, FESC, FICC, Department of Cardiology, Nizam's Institute of Medical Sciences, Hyderabad 500082, Telangana, India (e-mail: mail2jyotsna@rediffmail.com).
\end{abstract}

According to fluoro access study, even with the fluoro-guided FA puncture is an inappropriate site only in $20.4 \%$ access. Therefore, even fluoroscopic guidance is not good for the safe site of CFA puncture, especially in obese individuals. Even though fluoroscopically the needle direction is kept to puncture the artery at the inferior border of the head of the femur, depending on the depth of skin and subcutaneous tissue, this may puncture at a different $\operatorname{site}^{2}$ (-Fig. 2).

Ideally, puncture should be done under fluoroscopic or ultrasound guidance with the micropuncture needle. Sometimes it even requires femoral angiogram from the other route. Actual safe puncture site of the CFA should be done in every case, more so if there is a plan for vascular device closure. $^{3}$ The ideal site is below the interior border of the inferior epigastric artery. ${ }^{4}$

If high puncture of the CFA is done, even though the femoral head is there for compression, chances of retroperitoneal bleed are high. In low puncture of the CFA, hemostasis is difficult as there is no femoral head for proper compression.

- Ultrasound-guided femoral artery accesses: The major advantage with the ultrasound-guided versus palpation-guided access is the site of the exact site of puncture ${ }^{5}$ and avoidance of multiple attempts. ${ }^{6-10}$ For example, in SFA ostial occlusion case can feel the normal profunda femoris pulsations and we will puncture that artery. With ultrasound guided access, we can detect the SFA occlusion.

- Femoral artery puncture with micropuncture needlefluoroscopic guided: The micropuncture kit comes with a $21 \mathrm{G}$ needle, 0.018 -in guidewire, and 4 or $5 \mathrm{~F}$ micropuncture sheath, with a respective dilator for initial access and exchange to a 0.035 -in wire. After FA puncture with a microneedle, 0.18 wire is passed under fluoroscopy. This is very important as the wire may enter into small branches with our much resistance and can damage the vessel. This becomes the source for retroperitoneal bleed. The interface or needle to the wire gives where we punctured the CFA or not (-Fig. 3). If we are not sure, we can remove the needle, as it is the only micropuncture needle, and try once again. Otherwise, we can inject contrast directly through the needle (which is not very safe) or after through the dilator to confirm the needle position.
License terms

()(1) $\Theta \circledast$ 

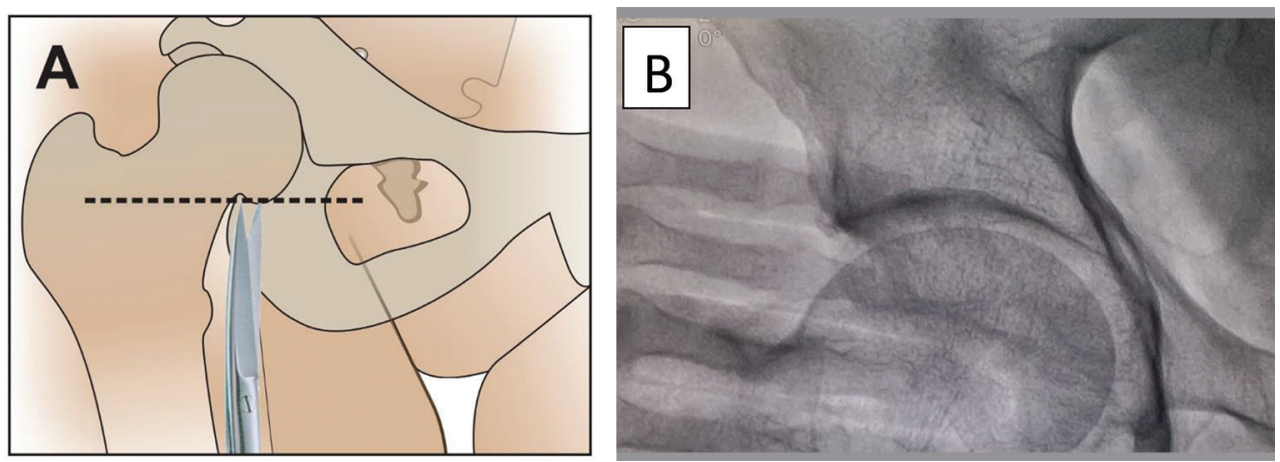

Fig. 1 (A) Anatomic landmarks and (B) fluoroscopic guidance of needle for FA puncture.

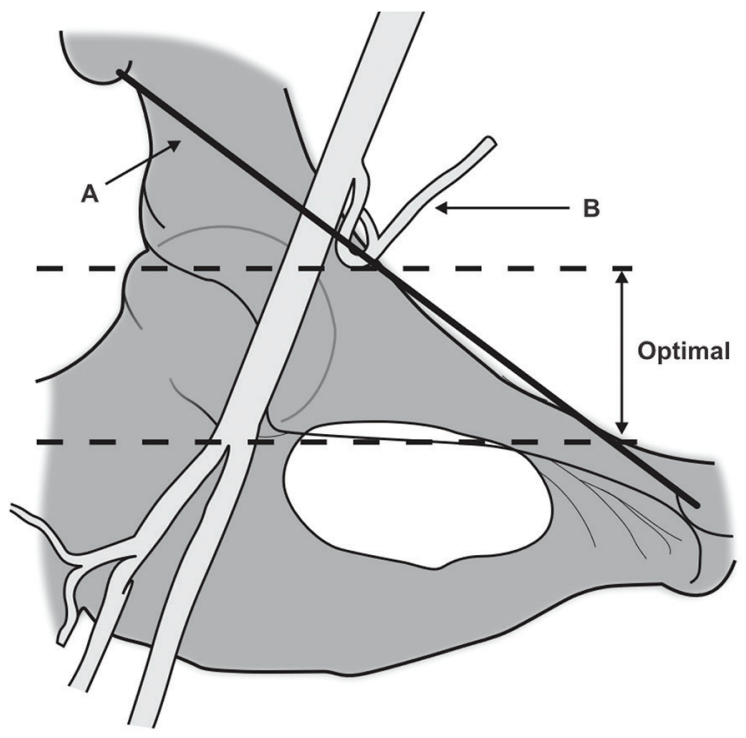

Fig. 2 The ideal site for puncture of CFA. A, inguinal ligament; $B$, inferior epigastric artery; optimal, in between the dashed lines (between the inferior border of the inferior epigastric artery and above the bifurcation).

\section{- Ultrasound-guided femoral access puncture with the} micropuncture needle: As we can see the CFA and its bifurcation along with the disease of other parts of the artery directly, this is a better and safer method for FA accesses. Besides, we can ensure that only the anterior wall of the artery is punctured without touching the posterior wall and also avoiding the venous puncture, so decreasing the chances of hematomas as well as arteriovenous (AV) fistulas. Other anomalies such as high bifurcations of CFA and dissection can be seen directly. Even advancement of the wire can be done under ultrasound guidance instead of fluoroscopy, by decreasing the radiation to both the operator and the patient. The same thing is proved in RAUST (Radial Artery access with Ultrasound Trial) study. ${ }^{7}$

- Common femoral artery access with the smart needle: Instead of using separate ultrasound for imaging, there are needles with Doppler signals at the tip of the needle. Smart needle (non-ultrasound-guided access techniques) from vascular solution company is of that variety. It detects the blood flow and gives an audio output. Therefore, we can differentiate arterial blood flow from the venous flow. As

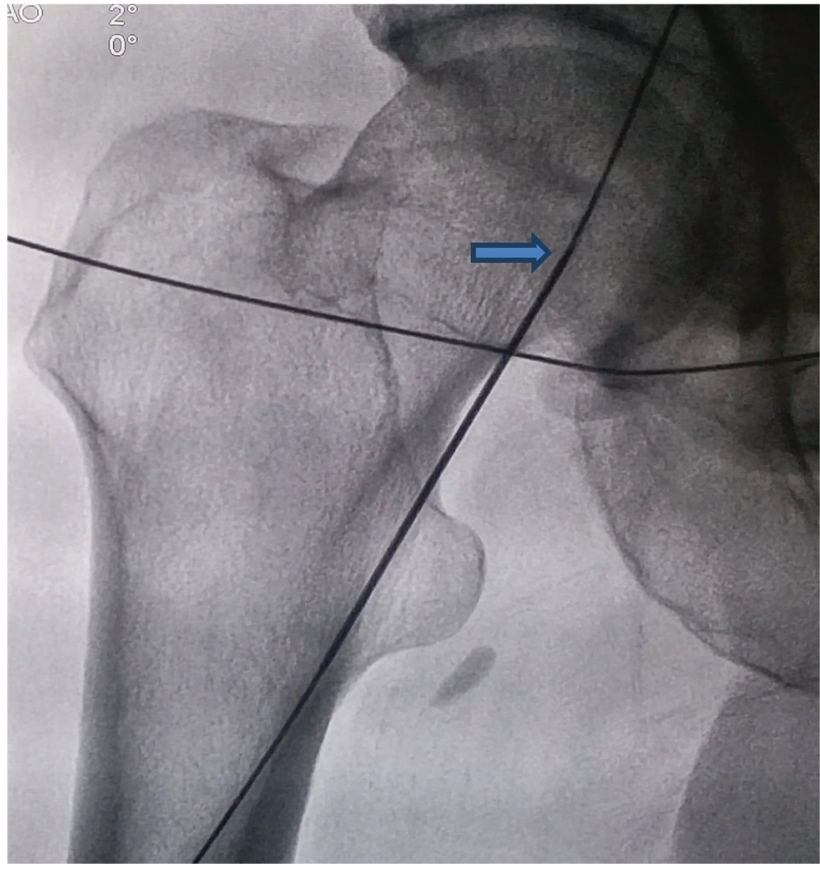

Fig. 3 The site of interface (blue arrow) between the needle and wire gives an idea of the site of CFA puncture.

the artery is an approached, audio sound increases. The major disadvantage is that we cannot differentiate the main artery from its branches.

\section{For Large Sheath Access}

For intra-aortic balloon pump (IABP), $7 \mathrm{~F}$ and Impella $8 \mathrm{~F}$ sheath is required. Very large arterial sheaths are required in a case of TAVI (transcatheter aortic valve implantation) or TEVAR (thoracic endovascular aortic repair). Then it is better to puncture with the microcatheter after confirming the artery as CFA; larger sheath can be upgraded. Otherwise, injection from the other femoral artery side is used as a roadmap to puncture the exact site on this side of CFA.

\section{Sheath Removal}

Adequate compression just proximal to the site of skin puncture for at least 30 minutes is ideal. Other than manual compression, there are a variety of devices for compression and vascular device closure. We are not describing these devices as this is beyond the scope of this article. 
As this large-bore sheath may compromise the distal blood flow to the limb, to maintain the perfusion of the limb, modified perfusion strategies should be followed.

For larger sheath removal, either per close device closure or balloon inflation before the puncture site from the other arterial access site should be done to get proper hemostasis.

The disadvantages of the femoral route are mainly because of the big-size vessel. At least 4 to 6 hours of bedrest is required after sheath removal. The bigger the sheath size and the more the anticoagulation or GP2B inhibitors, the more compression time at the puncture site and the longer the bedrest are required. Owing to prolonged bedrest, back discomfort and urinary retention may occur. Major complications are bleeding and hematomas that mainly depend on the low, high middle, and high punctures of the femoral artery. ${ }^{10}$ This high puncture may cause a retroperitoneal bleed. Others are pseudoaneurysm, AV fistulas, vessel damage and dissection, occlusion of the artery, and femoral nerve neuropathy. Occasionally cholesterol embolization, an infection, may happen.

The predictors of vascular complications include greater age, female sex, usage of glycoprotein IIb/IIIa inhibitors, intervention within 24 hours of thrombolysis, larger heparin doses, thienopyridines, high and low body mass index, duration or complexity of procedure, longer time to sheath removal, emergent priority, acute myocardial infarction, cardiogenic shock, renal failure, and peripheral vascular disease. ${ }^{11}$

Physical examination can distinguish between the complications (hematoma, pseudoaneurysm, and AV fistula). A hematoma is a painful bulging mass and may have pulsatility, but without bruit. Pseudoaneurysm is a pulsatile bulging painful mass with bruit. In acute AV fistula, there is no mass and the only bruit is present, but this has to distinguish from atherosclerotic narrowing of the vessel.

\section{Antegrade Puncture}

This method is used for interventions, distal to SFA (distal or mid-SFA, popliteal or infrapopliteal). In the prone position, patient legs should be under the image intensifier. Try to feel the maximal CFA pulse above the inguinal ligament; then antegrade puncture of CFA should be done. Sometimes the floppy abdomen may prevent the proper feeling of the pulse, which requires scratching and plastering of the abdomen. Immediately after puncturing of the femoral artery, when a good jet of blood flows back, check injection through the needle itself should be done to confirm that the needle tip is in the CFA, but not in profunda femoris. Then 0.35-in wire has to pass under fluoroscopy to secure the access.

\section{Radial Artery Access}

Campeau did first transradial cardiac catheterization in $1989 .{ }^{8}$ First transradial percutaneous coronary intervention (PCI) was done by Kiemeneij in 1993. Ideally, radial puncture should be done after performing Allen's test.

The steps in Allen's test are

- Empty the blood in the hand by fist and opening.

- Then compress both the radial and ulnar arteries.
- Release the compression on the ulnar artery; then see the time take to blush the hand.

Even though this test is very useful, the false-positive rate is high, especially in dark-skin individuals. Therefore, Barbeau's test is preferred to test the palmar arch patency. In this pulse, oximeter is used on the thumb while both arteries are being compressed. After releasing pressure on the ulnar artery, we may get four types of responses ( - Fig. 4 ).

- A-The pulse oximeter waveform remains normal with compression of the radial artery.

- B-The oximeter waveform decreases in magnitude (e.g., dampen).

- C-The oximeter waveform is obliterated with compression but returns within 2 minutes of the release of the radial artery.

- D-The oximeter waveform does not return within 2 minutes of the release of the radial artery.

\section{Radial Artery Puncture-Technique}

Complete puncture of the radial artery is required, as the artery is small caliber to ensure the position of the needle intraluminally. Even though complete puncture causes the posterior wall of vessel puncture, hematomas are not frequent, as the sheath during procedure and compression of the artery after the procedure can prevent the hematoma formation.

- Palpatory method: First to palpate the maximum impulse of the radial artery. Then puncture 2 to $3 \mathrm{~cm}$ proximal to

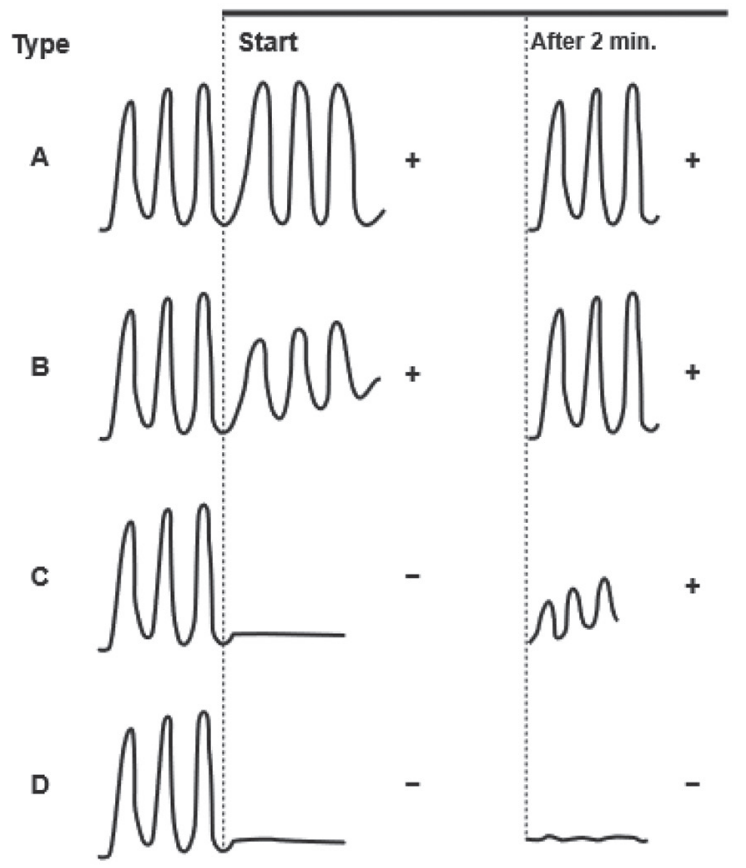

Fig. 4 Four (A-D) different responses by Barbeau's test (Adapted). After 2 minutes, the pulse wave 2 minutes after the release of compression on the artery; start, the pulse wave at the stating of the compression of the artery; Only Barbeau D patients should be excluded from transradial procedures. 
the flexion crease of the wrist. If the puncture is done more distally, reticulum may be encountered. In some cases, good radial artery pulse may be felt, but after the puncture, the blood flow may not be good. Then consider disease in the radial artery, or the pulse may be due to ulnar blood flow. Stabilize the radial artery in between the two fingers of the left hand, especially in elderly patients with atherosclerotic arteries that are very slippery.

- Ultrasound-guided puncture: To avoid the problems mentioned previously in the palpatory method, the ultrasound-guided puncture may be tried. The role of the ultrasound-guided puncture in radial artery access was uncertain until the RAUST was published. ${ }^{7}$

\section{Cannulation of Radial Artery after Puncture}

After puncturing the radial artery, 30 to $50 \mathrm{~cm}$ length, floppy straight or small angulation tip (rather than a J-tip) with rigid shaft wire is preferred to enter the artery. Sheaths used for radial artery cannulations are 5, 6, or 7F. Usually, radial artery size varies from 2 to $3 \mathrm{~mm}$, so big-size sheaths are not advisable through the radial route. Sheath length may be of 10 or $21 \mathrm{~cm}$. Some believe that longer sheaths prevent the radial spasm. Sheath with side port is better as it facilitates the delivery of drugs like the cocktail. These sheaths may be with single or double dilators. Double dilators have a tapered atraumatic tip for prevention of atrial trauma, but transition between the first and second dilators causes resistance at the skin insertion site. Standard guides used for the femoral route can be used through the radial route also, but in some cases, the right radial route may require short JL curve and/or longer JR curve. Other catheters and guides preferred for the radial route are Castillo curves and Sones catheters. Modified venous graft catheters and internal mammary arterial catheters can also be used through this route.

There are multiple advantages for the transradial route. Because of dual blood supply (both radial and ulnar arteries), hand ischemia is less. The patient can be ambulatory immediately. Patients with heart failure or any other cause of dyspnea or back pain may feel by this route. As it is easily accessible and compressible, the success of puncture and bleeding complications at the puncture site are less. Patient preference is also more with the radial route. This is an alternative route in patients with aortoiliac disease.

The disadvantages of the radial route are due to small caliber and tortuosity of the vessel. These are radial spasm and radial loops. In the early days of transradial procedures, the learning curve was steeper, but now most residents are starting with the radial route and are mastering radial artery puncture better than femoral artery puncture. Radial can accommodate smaller-size sheathes only; therefore, interventions requiring 7 or $8 \mathrm{~F}$ and above cannot be performed through the radial route. If the hand is kept parallel to the chest, cannulation of coronaries becomes easy. Usually, usage of guide manipulation requires more practice than the femoral route. A decade back it was said that radial route procedures take more fluoroscopic time with the increase in radiation dose to both the operator and the patient than the femoral route, but recent evidence is against it.

\section{Cocktail after Cannulation}

To prevent the radial spam and clot, cocktail containing nitroglycerin of $200 \mu \mathrm{g}, 5,000$ units of heparin along with $5 \mathrm{mg}$ of diltiazem should be given. This regimen can be altered in different situations. Like in severe left ventricular dysfunction and severe bradycardia, diltiazem may be avoided. In the case of severe aortic stenosis, it is better to avoid nitroglycerin.

\section{Right or Left Radial Artery}

Usually right radial artery is preferred over the left artery, as the comfort to the operator is better from the right radial route. After the left radial artery cannulation, the hand to be positioned on the abdomen and strain on the operator back is more.

In few circumstances, the left radial artery is preferred over the right radial route such as cannulation of left internal mammary artery graft, interventions through descending aorta (renal or aortoiliac angioplasty), and access of aortic branches. In short stature, obese individuals, the arch and descending aorta can be easily accessed through left radial route than the right radial route. In these patients even aorta in compressed longitudinally, so even cannulation of coronaries becomes difficult from the right radial route. According to 75 or $5 \mathrm{ft}-5$-in rule, with elderly patients aged $>75$ years or height $<5 \mathrm{ft} 5$ in $(165 \mathrm{~cm})$, it is better to do left radial puncture. Even though the tortuosity of subclavian and innominate artery known to occur on both side, it is less on the left side.

Usually, however, the right radial route right coronary artery (RCA) engagement is easy and the left radial route left main coronary artery (LMCA) cannulation is easy.

\section{Sheath Removal}

Whatever may be the activated clotting time (ACT), after the transradial procedure immediately, we can remove the sheath. Radial artery compression can be done manually or by a transradial band. In PROPHET (Prevention of Radial Artery Occlusion Patent Hemostasis Evaluation Trial), for better radial artery patency was there with patent hemostasis protocol than with traditional protocol. In the traditional group, transradial band was applied for 2 hours. In patent hemostasis group, after radial band application, reverse Barbeau's test was done, and compression pressure is adjusted until the plethysmographic signal of the radial flow establishes. At that compression pressure, the radial band was kept for 2 hours. ${ }^{12}$

The major complications following the radial artery cannulation are spasm and radial artery occlusion. The radial artery occlusion may occur temporarily due to significant spasm that may extend up to 24 hours. In first 24 hours, if radial pulse is not felt, ultrasound should be to rule out the thrombus. If thrombus is not there, low-dose nitroglycerin infusion should be given. If the thrombus is demonstrated, either conventional or low-molecular-weight heparin should be started. If the patient symptom of pain is not relieved even after 48 hours, it is better to do thrombectomy. Often, the radial artery occlusions are asymptomatic. The strategies to decrease the radial artery thrombosis are the use of smaller sheaths, use of sheathless guides, patent hemostasis, and anticoagulation along with the cocktail. 


\section{Snuffbox Puncture}

This is otherwise the puncture of distal radial artery puncture through the anatomical snuff box. This is mainly useful in where the upper limb movements are restricted such as frozen shoulder, elbow, or wrist. ${ }^{13,14}$

Puncture (based on pulse palpation) of the distal radial artery should be done using a 20G micropuncture needle or a cannula by the needle technique. In agreement with the MEMORY (Manual Versus Mechanical Compression of the Radial Artery After Transradial Coronary Angiography: The MEMORY Multicenter Randomized Trial) trial, for the forearm transradial access (TRA), manual hemostasis for the distal transradial access (dTRA) was significantly shorter than that for the mechanical one. ${ }^{15}$

\section{Vascular Complications of Radial Artery Access}

- Accelerated atherosclerosis

- AV fistula

- Avulsion of artery

- Bleeding

- Compartment syndrome

- Cutaneous infection

- Delayed reflex sympathetic dystrophy

- Digital ischemia

- Hematoma

- Radial artery occlusion

- Perforation, laceration, dissection

- Pseudoaneurysm

- Vessel spasm and vasovagal reaction

- Subcutaneous granulomatous reaction (hydrophilic coating)

- Subacute and delayed occlusion

- Infection

- Crossover to the femoral route-RIVAL (RadIal Vs femorAL access for coronary intervention) trial $^{6}$

\section{Ulnar Artery Access}

Ulnar cannulation facilitates preservation of radial artery for coronary artery bypass grafting (CABG). Before selecting the case for ulnar artery puncture, reverse Allen's test should be done. To cannulate this artery, puncture has to be 2 to $3 \mathrm{~cm}$ above the crease of the wrist. Complications for this vascular access are same as for radial except for severe vasospasm.

\section{Brachial Artery Access}

Sones and Shirey used the brachial route to do coronary angiography (CAG) in 1959.16

- Brachial artery puncture: Ideal site to puncture brachial artery is just proximal to the antecubital fossa. More precisely it can be done with fluoroscopic guidance over the olecranon process. To decrease the complication rate, it is better to use microcatheter under ultrasound guidance. During the procedure, to ensure the proper blood supply to the hand by monitoring with pulse oximeter is good along with clinical pain symptom. In children and neonates also, brachial route is preferred than radial route. ${ }^{17}$
If IABP 30-cc balloon is used through the brachial route, we have to do the procedure without the sheath. Besides, $5 \mathrm{~cm}$ covering sheath in proximal edge of the IABP also has to be peeled off to allow an adequate intra aortic position in the descending aorta. ${ }^{18}$

- Left versus right brachial access: Almost similar to the right and left radial. By brachial puncture rather than radial artery puncture, we will gain 10 to $15 \mathrm{~cm}$ more so even superficial and mid-SFA lesions can be treated. Though the left brachial route coronary cannulation is better with the Judkins catheters, Amplatz catheters are better with the right brachial route.

Complications for the brachial accesses include brachial artery occlusion, hematoma, dissection, pseudoaneurysm, median nerve compression, and Orator's hand posture.

\section{Subclavian Artery Access}

Early reports of using axillary artery as an access site for intervention were reported in the early 1990s. ${ }^{19}$ However, for TAVI procedure, surgical expose is preferred than a percutaneous puncture.

\section{Axillary Artery Access}

The femoral and iliac artery sizes depend on sex, patient size, and comorbidities. Even though the axillary artery is smaller than femoral $(\sim 6.5 \mathrm{~cm})$ because of the lesser incidence of atherosclerotic arterial stenosis, axillary artery access has become an alternative for large bore sheath..$^{20}$ This access can be considered not only in patients with PVD of femoral but also for TAVI and mechanical support devices. The advantage of using the axillary artery than femoral artery for mechanical support devices, especially in the bridge-to-transplant cases in which prolonged support is required, this route facilitates to ambulate the patient.

The axillary artery is punctured in between the second and third portions, at the lateral border of the pectoralis minor muscle with the arm in 90-degree abducted position. At this site, brachial plexus injury is less; pneumothorax occurs is less and is easily compressible. The puncture can be under ultrasound guidance or after the selective angiogram. Once the axillary artery and all branches are defined, the access point should be lateral to the thoracoacromial artery and medial to the circumflex humeral arteries. The precautions to be taken for axillary artery access is same as mentioned for large-bore sheath of the femoral artery.

\section{Popliteal Artery Access}

Popliteal artery access is mainly used for SFA occlusions when other access is not there. ${ }^{21-23}$ Knowledge of the anatomy of the popliteal artery is required for successful cannulation of the popliteal artery.

- Anatomy and technique of puncture: The popliteal artery is the extension of the SFA; it courses obliquely through the popliteal fossa from the upper medial border 
to just lateral to the midline at the lower end. It leaves the popliteal fossa and divides into the anterior tibial artery and the tibioperoneal trunk in the leg. The tibial nerve is the lateral most structure in the neurovascular bundle with the popliteal vein in the middle.

In the femorotibial popliteal space, the artery and vein are just anteroposterior, and artery puncture has to be done through the vein only ( $\mathbf{- F i g . 5}$ ). Therefore, puncture at this site causes AV fistulas very frequently. Six to $7 \mathrm{~cm}$ above the femorotibial joint space is ideal where the artery is medial to the vein. ${ }^{24}$

Usually, popliteal access is done in the prone position, even though few authors mention the supine position also. ${ }^{25}$ Puncture of the artery is done with ultrasound guidance or roadmap technique from the opposite femoral site with the micropuncture needle or standard $18 \mathrm{G}$ needle. In this roadmap technique, we can visualize the artery only, and we cannot know the position of the vein. Again the same problem of AV fistula may arise in this technique. It is better to use small-size sheaths 4 or $5 \mathrm{~F}$, prevent the complications, and upsize only when it is required.

- Anticoagulation: Periprocedural anticoagulation differs from other puncture site anticoagulation regimen, as this may require antegrade puncture of the femoral artery again. Therefore, the dose is adjusted to case-to-case basis; usually 2,000 to 5,000 units of unfractionated heparin are recommended.

Even though some reported distal embolization and arterial spasm in popliteal artery puncture, practical experience does not substantiate that.

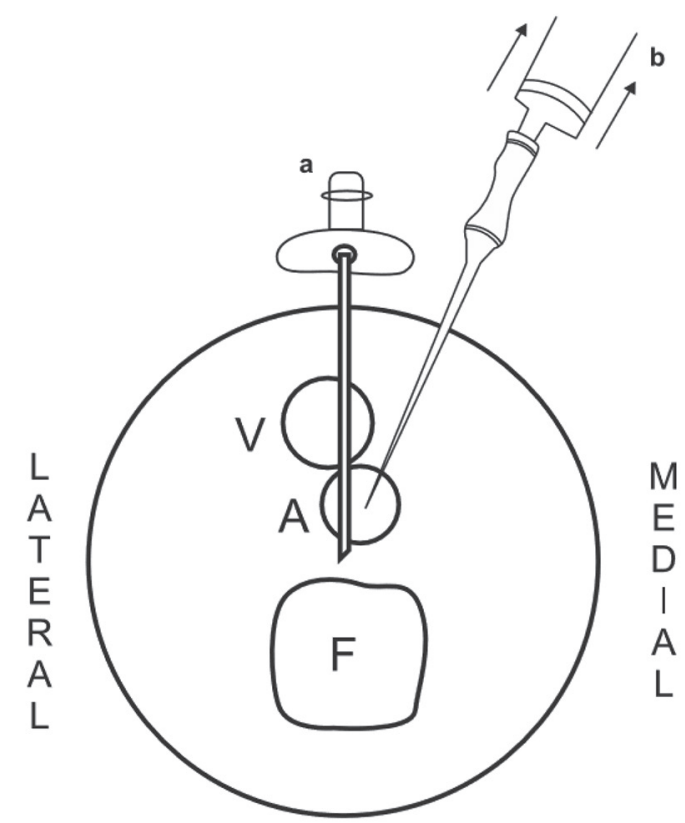

Fig. 5 By Seldinger's technique (the needle position is represented as "a"), AV fistulas are common, whereas when the needle is positioned from medial to lateral, avoiding the vein (b) is the correct way to puncture the popliteal artery (Adopted) A, artery; F, femur; V, vein.

\section{Pedal Artery Access}

These accesses can be used when there is at least $5 \mathrm{~cm}$ of runoff length of the vessel. When this is only patent artery for distal runoff, it is better to avoid that pedal artery antegrade puncture, as it precipitates the ischemia of the foot. If there is no other option than puncturing that pedal artery only, it better do the procedure without the sheath. A puncture site 1 to $2 \mathrm{~cm}$ proximal to the level of the medial malleolus is recommended for the posterior tibialis artery, whereas the dorsalis pedis artery is most commonly punctured 1 to $3 \mathrm{~cm}$ distal to the level of the malleoli. These sites are preferred mainly for proper compression to achieve homeostasis. Puncture can be done by ultrasound guidance or with roadmap. ${ }^{26}$ For dorsalis pedis artery, puncture of the foot should be in the neutral position with slight dorsiflexion. For posterior tibial artery, puncture of the foot should in the lateral position with slight abduction. Like the radial artery, to prevent the vasospasm, $200 \mathrm{mg}$ of nitroglycerin or $2.5 \mathrm{mg}$ of verapamil needs to be given. The same radial band can be used instead of manual compression to achieve hemostasis in these pedal arteries.

\section{Arterial Access via a Bypass Graft}

If there is no access, bypass graft can be used cautiously as the access site complications with graft access are very high ( 12\%). In a hemodialysis patient, venous obstructions are better done through the AV fistula site than from the retrograde venous route.

\section{Vascular Access in Pediatrics}

The same access as in adults can be tried. In addition in neonates, umbilical vessels can be used. The sheath size is to determine by the estimated size of the femoral artery for that age group children. Inappropriate big size of sheath usage causes the frequent loss of the pulse and limb ischemia in children.

\section{Direct Aorta Access}

For TAVI, direct aortic access is used, but that access is through surgical approach. In initial catheterization era, direct aortic puncture from the lumbar area was mentioned historically, but never used subsequently.

\section{Transcaval Aortic Access}

For TAVI and aortic aneurysm, transcaval aortic access is tried $^{27}$ when conventional access routes are not suitable. The proximity of the caudal aorta and inferior vena cava (IVC) facilitated this type of approach. There should be no mural aortic calcification for this procedure. With intravascular ultrasound or conventional simultaneous both arteriogram and venogram, the puncture is to be done from IVC to the aorta. After delivery of the TAVI valve or TEVAR device, the track has to close with closure devices. 


\section{Carotid Artery Puncture-Percutaneous Common Carotid Artery}

Puncture of the percutaneous common carotid artery (PCA) is done for cardiac catheterization for pediatric patients. ${ }^{28}$ In low-weight children, for interventions such as aortic valvuloplasty or coarctation dilatation or BT shunt dilatations, transcarotid route was chosen in 45 children instead of the femoral route. The preferred site for the common carotid artery was well below the carotid bifurcation, mostly, midway between the mandible and clavicle, under ultrasound guidance. Heparinization after sheath insertions was in the dose of 50 to $100 \mathrm{U} / \mathrm{kg}$. Manual compression was done after the sheath removal for hemostasis.

Strictly speaking, transcarotid artery revascularization (TCAR) is not a percutaneous procedure, but it is done with en route transcarotid neuroprotection and stent system, through a minimally invasive incision for the cases where endarterectomy or stenting in carotid disease is difficult.. ${ }^{29}$

\section{Left Heart Access}

- From the cardiac apex

- Though interatrial septum

This route is mentioned for completion sake, but this is not directly the puncture of an artery.

\section{Conflict of Interest}

None.

\section{References}

1. Arterial and Venous Access in the Cardiac Catheterization Lab. In: Abu-Fadel M, ed. Contributions by Abu-Fadel M, Farah F, Hawkins B, et al. Rutgers University Press Medicine; June 28, 2016

2. Abu-Fadel MS, Sparling JM, Zacharias SJ, et al. Fluoroscopy vs. traditional guided femoral arterial access and the use of closure devices: a randomized controlled trial. Catheter Cardiovasc Interv 2009;74(4):533-539

3. Sandoval Y, Burke MN, Lobo AS, et al. Contemporary arterial access in the cardiac catheterization laboratory. JACC Cardiovasc Interv 2017;10(22):2233-2241

4. Sobolev M, Slovut DP, Lee Chang A, Shiloh AL, Eisen LA. Ultrasound-guided catheterization of the femoral artery: a systematic review and meta-analysis of randomized controlled trials. J Invasive Cardiol 2015;27(7):318-323

5. Rafie IM, Uddin MM, Ossei-Gerning N, Anderson RA, Kinnaird TD. Patients undergoing PCI from the femoral route by default radial operators are at high risk of vascular access-site complications. EuroIntervention 2014;9(10):1189-1194

6. Jolly SS, Yusuf S, Cairns J, et al; RIVAL trial group. Radial versus femoral access for coronary angiography and intervention in patients with acute coronary syndromes (RIVAL): a randomised, parallel group, multicentre trial. Lancet 2011;377(9775):1409-1420

7. Seto AH, Roberts JS, Abu-Fadel MS, et al. Real-time ultrasound guidance facilitates transradial access: RAUST (Radial Artery access with Ultrasound Trial). JACC Cardiovasc Interv 2015;8(2):283-291
8. Shiloh AL, Savel RH, Paulin LM, Eisen LA. Ultrasound-guided catheterization of the radial artery: a systematic review and meta-analysis of randomized controlled trials. Chest 2011;139(3):524-529

9. Agostoni P, Biondi-Zoccai GG, de Benedictis ML, et al. Radial versus femoral approach for percutaneous coronary diagnostic and interventional procedures; systematic overview and meta-analysis of randomized trials. J Am Coll Cardiol 2004;44(2):349-356

10. Sherev DA, Shaw RE, Brent BN. Angiographic predictors of femoral access site complications: implication for planned percutaneous coronary intervention. Catheter Cardiovasc Interv 2005;65(2):196-202

11. Bhatty S, Cooke R, Shetty R, Ion SJ. Femoral vascular access-site complications in the cardiac catheterization laboratory: diagnosis and management. Interv Cardiol (Lond) 2011;3(4):503-514

12. Pancholy S, Coppola J, Patel T, Roke-Thomas M. Prevention of radial artery occlusion-patent hemostasis evaluation trial (PROPHET study): a randomized comparison of traditional versus patency documented hemostasis after transradial catheterization. Catheter Cardiovasc Interv 2008;72(3):335-340

13. Ziakas A, Koutouzis M, Didagelos M, et al. Right arm distal transradial (snuffbox) access for coronary catheterization: initial experience. Hellenic J Cardiol 2018;S1109-9666(18)30379-8

14. Petroglou D, Didagelos M, Chalikias G, et al. Manual versus mechanical compression of the radial artery after transradial coronary angiography: the MEMORY multicenter randomized trial. JACC Cardiovasc Interv 2018;11(11):1050-1058

15. Davies RE, Gilchrist IC. Back hand approach to radial access: the snuff box approach. Cardiovasc Revasc Med 2018;19(3 Pt B):324-326

16. Sones FM Jr, Shirey EK. Cine coronary arteriography. Mod Concepts Cardiovasc Dis 1962;31:735-738

17. Schindler E, Kowald B, Suess H, Niehaus-Borquez B, Tausch B, Brecher A. Catheterization of the radial or brachial artery in neonates and infants. Paediatr Anaesth 2005;15(8):677-682

18. Golwala H, Zacharias S, Latif F. Bilateral arm approach for percutaneous coronary intervention of unprotected left main supported by a modified intra-aortic balloon pump. J Invasive Cardiol 2012;24(4):183-184

19. Andros G, Harris RW, Dulawa LB, Oblath RW, Schneider PA. Subclavian artery catheterization: a new approach for endovascular procedures. J Vasc Surg 1994;20(4):566-574, discussion 574-576

20. Kaki A, Alraies MC, Blank N, ET AL. TCT-757 axillary artery: access for mechanical circulatory support devices in patients with prohibitive peripheral arterial disease presenting with cardiogenic shock. J Am Col Cardiol 2018;72(Suppl 13):B302B303. DOI: 10.1016/j.jacc.2018.08.1983 [epub ahead of print]

21. Noory E, Rastan A, Schwarzwälder U, et al. Retrograde transpopliteal recanalization of chronic superficial femoral artery occlusion after failed re-entry during antegrade subintimal angioplasty. J Endovasc Ther 2009;16(5):619-623

22. Spinosa DJ, Harthun NL, Bissonette EA, et al. Subintimal arterial flossing with antegrade-retrograde intervention (SAFARI) for subintimal recanalization to treat chronic critical limb ischemia. J Vasc Interv Radiol 2005;16(1):37-44

23. Fanelli F, Lucatelli P, Allegritti M, Corona M, Rossi P, Passariello R. Retrograde popliteal access in the supine patient for recanalization of the superficial femoral artery: initial results. J Endovasc Ther 2011;18(4):503-509

24. Trigaux JP, Van Beers B, De Wispelaere JF. Anatomic relationship between the popliteal artery and vein: a guide to accurate angiographic puncture. AJR Am J Roentgenol 1991;157(6):1259-1262 
25. Kawarada O, Yokoi Y. Retrograde 3-French popliteal approach in the supine position after failed antegrade angioplasty for chronic superficial femoral artery occlusion. J Endovasc Ther 2010;17(2):255-258

26. Spinosa DJ, Leung DA, Harthun NL, et al. Simultaneous antegrade and retrograde access for subintimal recanalization of peripheral arterial occlusion. J Vasc Interv Radiol 2003;14(11):1449-1454

27. Uflacker A, Lim S, Ragosta M, et al. Transcaval aortic access for percutaneous thoracic aortic aneurysm repair: initial human experience. J Vasc Interv Radiol 2015;26(10):1437-1441
28. Justino H, Petit CJ. Percutaneous common carotid artery access for pediatric interventional cardiac catheterization. Circ Cardiovasc Interv 2016;9(4):e003003

29. Guimaraens L, Theron J, Casasco A, Cuellar H. Carotid artery stenting by direct percutaneous puncture. J Vasc Surg 2011;54(1):249-251 